\title{
Perfil bioquímico e capacidade antioxidante total em cavalos de polo suplementados com selênio e vitamina-E
}

\author{
Biochemical profile and total antioxidant capacity in polo horses \\ supplemented with selenium and vitamin-E
}

\author{
Waldsylvio Silva VieiraI Isabella Manes Soutto Mayor da Motta Rodrigues" \\ Natália Pereira Lopes Frade ${ }^{\mathrm{II}}$ Cristiane Divan Baldani ${ }^{\mathrm{IV}}$ \\ Rita de Cassia Campbell Machado Botteon ${ }^{\text {IV }}$ \\ Paulo de Tarso Landgraf Botteon ${ }^{\mathrm{IV}^{*}}$
}

RESUMO

O objetivo deste trabalho foi avaliar as alterações bioquímicas e da capacidade antioxidante total (CAT) em cavalos de pólo, e o efeito da suplementação de selênio (Se) e vitamina E (Vit-E) sobre esses parâmetros. Dezesseis equinos aleatoriamente divididos em dois grupos, GE/Se (suplementados com Vit-E e Se) e GC (grupo controle), participaram de um jogo de polo com quatro períodos (chukkas) de 7 minutos. Cada cavalo competiu em dois chukkas, com 7 minutos de intervalo. Amostras de sangue foram colhidas $15 \mathrm{~min}$ antes do jogo (basal), ao final do 1ㅇ e 2o chukkas e 24h, 48h e $72 \mathrm{~h}$ após o término da competição. Foram analisadas as atividades séricas da aspartato aminotrasferase (AST), creatinoquinase (CK) e lactato desidrogenase (LDH), a glicemia e lactatemia e a capacidade antioxidante total (CAT). Os valores de aspartato aminotrasferase, creatinoquinase e lactato desidrogenase não apresentaram diferenças significativas entre o GE/Se e o GC. A atividade de AST e LDH aumentou significativamente em relação ao valor basal $(P=0,097)$ e $(P=0,0002)$, respectivamente, e permaneceram elevadas por mais tempo no GC. A CK não apresentou variações significativas em relação ao basal em ambos os grupos. A lactatemia aumentou significativamente após cada chukka, comparada ao basal e foi maior no GC que no GE/Se após o primeiro chukka $(P<0,05)$. A CAT foi significativamente menor $\left(P=2,90^{-25}\right)$ após o primeiro chukka, retornando aos valores basais após 24 horas, porém sem diferença entre os grupos. Conclui-se que a suplementação com vit-E e Se não influencia nos parâmetros avaliados. O exercício alterou o perfil bioquímico e a CAT sem causar lesões, sugerindo um bom condicionamento físico e adequada proteção antioxidante nos animais estudados.

Palavras-chave: antioxidantes, equino, polo.

\section{ABSTRACT}

The aim of this study was to evaluate the biochemical and the total antioxidant capacity (TAC) on polo horses, and the effect of selenium (Se) and vitamin E (vit-E) on these parameters. Sixteen horses were randomly divided into two groups, GE/ Se (supplemented with vit-E and Se) and GC (control group) participated in a game of polo with four periods (chukkas) of 7 min. Each horse has competed in two chukkas, with an interval of $7 \mathrm{~min}$. Blood samples were collected 15min. before the game (baseline), at the end of the $1^{\text {st }}$ and $2^{\text {nd }}$ chukkas and $24 \mathrm{~h}, 48 \mathrm{~h}$ and $72 \mathrm{~h}$ after the competition. Were analyzed the serum activity of aspartate aminotrasferase (AST), creatine kinase (CK) and lactate dehydrogenase (LDH), blood glucose and blood lactate concentration and total antioxidant capacity (TAC). There was no significant difference between GE/Se and GC to AST, CK and $L D H$. The activity of AST and $L D H$ increased significantly compared to the baseline value, $(P=0.097)$ and $(P=0.0002)$, respectively, and remain elevated for more time in GC. CK didn't change significantly in relation to baseline in both groups. The blood lactate was significantly higger after each chukka than in baseline, but don't heve significant difference between GE/Se and CG. The CAT decreased significantly $\left(P=2.90^{-25}\right)$ after the first chukka, returning to baseline in 24 hours, but there was no difference between groups. It is concluded that supplementation with vit-E and Se had no influence on the parameters evaluated. The exercise changed the biochemical profile and the TAC without causing injuries, suggesting a good conditioning and adequate antioxidant protection.

Key words: antioxidants, equine, polo.

\section{INTRODUÇÃO}

O estresse oxidativo (EO) ocorre quando existe uma maior ação dos agentes oxidantes frente aos antioxidantes (DEATON \& MARLIN, 2003). Fatores como dieta, idade, ingestão de fármacos, poluição,

\footnotetext{
IExército Brasileiro, $2^{\circ}$ Regimento de Cavalaria de Guarda, Rio de Janeiro, RJ, Brasil.

IIPrograma de Pós-graduação em Medicina Veterinária, Universidade Federal Rural do Rio de Janeiro (UFRRJ), Seropédica, RJ, Brasil.

IIIMédica Veterinária, Seropédica, RJ, Brasil.

${ }^{\text {IV }}$ Departamento de Medicina e Cirurgia Veterinária (DMCV), Instituto de Veterinária (IV), UFRRJ, 23890-000, Seropédica, RJ, Brasil.

E-mail: paulobotteon@gmail.com.*Autor para correspondência. 
umidade relativa e temperatura ambiente impróprios podem favorecer a ocorrência de EO (LIU et al., 2000). Essa situação traduz-se na incapacidade de impedir ou reparar os danos provocados por espécies reativas de oxigênio (ERO) sobre as estruturas celulares, mesmo em repouso (MOTA et al., 2004). Aumentos do EO ocorrem devido a elevação das EROs, pela redução da capacidade antioxidante ou pela conjunção desses fatores (DEATON \& MARLIN, 2003).

$\mathrm{O}$ exercício físico induz à diversas adaptações fisiológicas e bioquímicas ao cavalo. O incremento do metabolismo e captação de oxigênio pode induzir ao EO. O alto consumo de oxigênio durante esforço extenuante promove vantagens metabólicas na produção de energia, mas favorece a ocorrência de EO nas células musculares (HARGREAVES et al. 2002). O consumo de oxigênio durante o exercício pode aumentar 10 a 20 vezes no homem e 30 vezes no cavalo. Isso faz do cavalo ótimo modelo para se estudar o EO (WILLIAMS, 2003). Os oxidantes são capazes de lesar DNA, lipídeos e proteínas, porém, existem sistemas complexos de antioxidantes enzimáticos e não enzimáticos para proteger o organismo contra o EO. Os principais antioxidantes não enzimáticos são vitamina $\mathrm{C}$, vitamina $\mathrm{E}$, glutationa e ácido úrico (FERREIRA et al., 2008).

Cavalos de polo, além do esforço metabólico desenvolvido durante as arrancadas, estão sujeitos a lesões decorrentes das mudanças bruscas de direção e velocidade. Essas particularidades podem expor esses animais a situações de estresse que favoreceriam a ocorrência de EO. A proposta deste trabalho é avaliar as alterações bioquímicas e oxidativas induzidas pelo exercício em cavalos de polo e o efeito da suplementação com Vit-E e Se.

\section{MATERIAL E MÉTODOS}

Foram utilizados 16 equinos, clinicamente sadios, 11 machos castrados e cinco fêmeas, membros da equipe de polo do 2으, RCG, Regimento Andrade Neves - Exército Brasileiro, Rio de Janeiro/ RJ, mestiços, com idade média de 7,3 $\pm 2,3$ anos, peso médio de $446 \pm 15 \mathrm{~kg}$, escore corporal entre 4,5 e 5,0 (HENNEKE et al., 1983), estabulados em baias individuais, exercitados seis dias por semana e alimentados com $5 \mathrm{~kg}$ dia ${ }^{-1}$ de ração concentrada, $4 \mathrm{~kg}$ dia $^{-1}$ de feno de Coast-cross e $30 \mathrm{~g}$ de mistura mineral ${ }^{\mathrm{b}}$ (12UI dia ${ }^{-1}$ de vit-E e $0,36 \mathrm{mg} \mathrm{dia}^{-1}$ de Se ).

$\mathrm{O}$ estudo foi conduzido em delineamento inteiramente casualizado com dois tratamentos (tratado e controle) e oito repetições. $\mathrm{O}$ grupo tratado (GE/Se) recebeu suplementação oral com $20 \mathrm{~mL}$ de suplemento com vit-E e $\mathrm{Se}^{\mathrm{c}}$ (2300UI dia ${ }^{-1}$ de vit-E e 0,56 $\mathrm{mg} \mathrm{dia}^{-1} \mathrm{de} \mathrm{Se}$ ), por 20 dias antes do experimento, conforme recomendação do fabricante para cavalos com peso entre 400 e $500 \mathrm{~kg}$; e GC (grupo controle). Os grupos participaram de um jogo de polo que consistiu em quatro períodos (chukkas) de 7 minutos. Cada cavalo competiu em dois chukkas, com intervalo de 7 minutos. Após cada chukka, os animais foram examinados clinicamente, quando se observaram, à inspeção, sinais de claudicação; à palpação, sinais de lesões musculares ou lombares; e avaliados os parâmetros vitais: coloração de mucosas, por inspeção; o tempo de preenchimento capilar, por compressão digital, seguida de observação do tempo em segundos de reestabelecimento da vascularização; o turgor cutâneo, determinado por palpação; a temperatura retal foi aferida por termômetro digital; frequência cardíaca, frequência respiratória, murmúrio vesicular e motilidade intestinal, por auscultação.

As coletas de sangue foram realizada em seis momentos: M1 - 15min antes do início da prova, com os animais já encilhados; M2 e M3 colhidos após cada chukka; M4, M5 e M6, colhidos 24, 48 e 72 horas, após o início da prova, respectivamente. As amostras de sangue foram colhidas por punção da veia jugular, com tubos para coleta de sangue à váculo ${ }^{c}$ de $4 \mathrm{~mL}$, contendo ou não anticoagulante (EDTA, heparina ou fluoreto de sódio + EDTA). As amostras foram resfriadas em recipiente isotérmico com gelo seco. Em seguida, foram centrifugadas (5000rpm $3 \mathrm{~min}^{-1}$ ) para obtenção de soro e plasma, e novamente resfriadas e armazenadas em freezer $-20^{\circ} \mathrm{C}$. Amostras de sangue colhidas com fluoreto de sódio, foram empregadas na determinação das concentrações plasmáticas de lactato, segundo metodologia descrita por KOBAYASHI (2007). Amostras com EDTA foram utilizadas para a determinação do hemograma completo em contador automático de células sanguíneas ${ }^{\mathrm{d}} \mathrm{e}$ a concentração de proteínas plasmáticas totais por refratometria. A glicemia plasmática foi determinada a partir das amostras contendo fluoreto de sódio + EDTA, empregando-se kit comercial ${ }^{\mathrm{e}}$ (método enzimático colorimétrico). Nas amostras de soro, foram realizadas as determinações das concentrações de creatinina (método de ponto final), atividade sérica das enzimas AST, LDH e CK (método cinético) em analisador bioquímico automatizado ${ }^{\text {f }}$. Para avaliação da capacidade antioxidante total (CAT), parâmetro que representa o resultado das interações entre cada um dos componentes antioxidantes do soro, foram utilizadas amostras de plasma, armazenadas em freezer $-80^{\circ} \mathrm{C}$, até o momento da análise, através de kit comercials, conforme as recomendações do 
fabricante. As leituras foram efetuadas em leitor de microplacas $^{\mathrm{h}}$ a $405 \mathrm{~nm}$.

Os dados foram analisados quanto à distribuição normal pelo teste Shapiro-Wilk. Em seguida, foram submetidos ao teste Anova para medidas repetidas (RM-ANOVA). No pós-teste, para comparação múltipla de médias, foi aplicado o teste de Bonferroni, com 95\% de significância.

\section{RESULTADOS E DISCUSSÃO}

Não foram observadas alterações clínicas significativas nas avalições realizadas e os resultados foram condizentes com o nível de condicionamento dos animais. A dose de vit-E empregada foi cerca de três vezes maior que o nível recomendado: $80 \mathrm{UI} \mathrm{kg}^{-1}$ (NRC 1989). SICILIANO et al. (1997) observaram efeito protetor da vit-E com dose de $300 \mathrm{UI} \mathrm{kg}^{-1} \mathrm{MS}$. Para um consumo de $2 \% \mathrm{PV}$, um cavalo de $450 \mathrm{~kg}$ recebeu $2700 \mathrm{UI}$ dia $^{-1}$ de vit-E, dose empregada neste experimento, porém, os efeitos da vit-E só foram evidenciados a partir de 30 dias de suplementação. DUBERSTEIN (2009) administrou 3000UI dia ${ }^{-1}$, por 8 semanas, e concluiu que a vit-E pode minimizar os efeitos do EO. No Brasil, doses de 1000UI dia ${ }^{-1}$ durante 15 dias (YONEZAWA, 2010) e 2500UI dia ${ }^{-1}$ por 35 dias (DIAS et al., 2009) não evidenciaram efeitos da suplementação de vit-E.

A necessidade diária de Se para cavalos adultos é estimada em $0,1 \mathrm{mg} \mathrm{kg}^{-1}$ MS (NRC 1989). A dose utilizada neste estudo foi de $3,75 \mathrm{mg}$ animal $^{-1}$ $\mathrm{dia}^{-1}$, cerca de quatro vezes a necessidade para um consumo de $9 \mathrm{~kg} \mathrm{dia}^{-1}$ de $\mathrm{MS}$ e dose similar à empregada por RICHARDSON et al. (2006). Outros autores relataram o aumento da atividade da enzima glutationa peroxidase (GSx) após 30 dias de adição de Se (MOORE \& KOHN, 1991). Cavalos com deficiência de Se, dependendo da taxa de renovação de eritrócitos, apresentaram elevação de GSx 30 a 60 dias após o início da suplementação (ARAYA et al., 2004).

Lisina e magnésio presentes no suplemento não influenciam em adultos e nos parâmetros estudados neste experimento (VAN DEN HOVEN et al., 2011; RICH \& BREUER, 2002).

A análise conjunta da atividade sérica das enzimas musclares permitiu monitorar a ocorrência de dano muscular. Neste estudo, verificamos elevação significativa após o exercício em relação ao basal da atividade sérica das enzimas AST $(\mathrm{P}=0,0097)$ apenas no grupo controle e $\mathrm{LDH}(\mathrm{P}=0,0002)$ em ambos os grupos. Os valores de AST retornaram aos níveis basais às $24 \mathrm{~h}$ e a LDH permaneceu com valores elevados até $48 \mathrm{~h}$ (Tabela 1). Não houve diferença significativa entre os grupos, para ambas as enzimas ( $\mathrm{P}=0,3517$ para $A S T$ e $\mathrm{P}=0,5383$ para $\mathrm{LDH})$. O pico da atividade sérica de AST foi de 273,9UI L-1 , observado após o segundo tempo de jogo no grupo suplementado e $254,7 \mathrm{UI} \mathrm{L}^{-1}$ no grupo controle. DIAS et al. (2009) descreveram que cavalos de salto suplementados com vitamina E e selênio apresentaram valores de AST significativamente maiores nos animais não suplementados, e retorno aos valores basais seis horas após o exercício. O mesmo comportamento foi observado neste estudo, com valores maiores no grupo suplementado, porém sem diferença significativa em relação ao controle. SICILIANO et al. (1997) relatam o pico de AST ocorrendo 6 a 24h após o exercício, e retorno aos níveis basais $72 \mathrm{~h}$ após o exercício. Neste estudo, observamos valores máximos logo após o primeiro tempo de jogo no grupo controle e após o segundo tempo de jogo no grupo suplementado. Em ambos os grupos, o retorno aos níveis basais ocorreu $72 \mathrm{~h}$ após o exercício, assemelhando-se ao relatado por SICILIANO et al. (1997).

Em relação à atividade sérica de $\mathrm{LDH}$, o pico de atividade ocorreu após o segundo tempo de jogo para o $\mathrm{GE} / \mathrm{Se}\left(471,3 \mathrm{mg} \mathrm{dL}^{-1}\right)$, enquanto que, para o GC, o pico ocorreu $24 \mathrm{~h}$ após o jogo $\left(433,2 \mathrm{UI} \mathrm{L}^{-1}\right)$ (Tabela 1). Valores inferiores a $350 \mathrm{UI}$ $\mathrm{L}^{-1}$ são considerados normais para cavalos em treinamento (CORREA et al., 2010), como observado neste trabalho $72 \mathrm{~h}$ após o jogo. DIAS et al. (2009) observaram, em cavalos de salto, índices inferiores aos deste trabalho, com a tendência de recuperação mais rápida dos animais suplementados com vit-E e Se, que o GC, semelhante ao observado neste trabalho. Já THOMASSIAN et al. (2007) avaliaram equinos submetidos ao teste padrão de exercício progressivo em esteira e observaram picos de até $634,8 \mathrm{UI} \mathrm{L}^{-1}$.

$\mathrm{A}$ atividade sérica da $\mathrm{CK}$ não apresentou variações significativas no período avaliado ( $\mathrm{P}=0,3023)$, com valores basais de $230,3 \mathrm{UI} \mathrm{L}^{-1}$ para o GE/Se e 196,5UI L-1 para o GC; e picos de 302,2UI $\mathrm{L}^{-1}$ às $24 \mathrm{~h}$ para o GE/Se e $295,8 \mathrm{UI} \mathrm{L}^{-1}$ para o GC. Os valores da $\mathrm{CK}$ para animais em repouso foram maiores que os citados por FRANCINATO et al. (2006); KOWAL et al. (2006); DIAS et al. (2009); CAIADO et al. (2011); e MICHIMA et al. (2011), possivelmente, devido à rotina diária de treinamento desses equinos. Tais resultados foram semelhantes aos observados por RIBEIRO et al. (2004) e THOMASSIAN et al. (2007). Nesses estudos, os autores relataram valores de 88 a $362 \mathrm{UI} \mathrm{L}^{-1}$ após a atividade física. VOLFINGER et al. (1994) calcularam a magnitude da lesão muscular em cavalos, baseada na CK liberada do músculo esquelético, e concluíram que 
Tabela 1 - Parâmetros bioquímicos expressos em médias \pm desvios-padrão em equinos de polo antes do jogo (Zero), após o primeiro e segundo tempos ( $1^{\circ} \mathrm{T}$ e $\left.2^{\circ} \mathrm{T}\right)$, e $24 \mathrm{~h}, 48 \mathrm{~h}$ e $72 \mathrm{~h}$ após o jogo, nos grupos suplementados com vitamina E e selênio (GE/Se, $\left.\mathrm{n}=8\right)$ e controle $(\mathrm{GC}, \mathrm{n}=8)$.

\begin{tabular}{|c|c|c|c|c|c|c|c|}
\hline Parâmetros & Grupos & Zero & $1^{\circ} \mathrm{T}$ & $2^{\circ} \mathrm{T}$ & $24 \mathrm{~h}$ & $48 \mathrm{~h}$ & $72 \mathrm{~h}$ \\
\hline AST & $\mathrm{GE} / \mathrm{Se}$ & $243,9 \pm 48,7 \mathrm{a}$ & $272,1 \pm 26,2 \mathrm{a}$ & $273,9 \pm 27,0 \mathrm{a}$ & $251,0 \pm 46,2 \mathrm{a}$ & $251,3 \pm 35,7 \mathrm{a}$ & $230,7 \pm 49,4 \mathrm{a}$ \\
\hline$\left(\mathrm{UI} \mathrm{L}^{-1}\right)$ & GC & $211,9 \pm 46,7 \mathrm{a}$ & $254,7 \pm 36,6 b$ & $250,8 \pm 30,8 b$ & $236,6 \pm 23,3 \mathrm{ab}$ & $247,1 \pm 38,9 a b$ & $219,3 \pm 39,3 \mathrm{ab}$ \\
\hline $\mathrm{LDH}$ & $\mathrm{GE} / \mathrm{Se}$ & $341,8 \pm 98,6 \mathrm{a}$ & $409,5 \pm 55,7 b$ & $471,3 \pm 75,3 b$ & $425,3 \pm 72,0 \mathrm{ab}$ & $396,7 \pm 66,3 \mathrm{a}$ & $329,2 \pm 51,4 \mathrm{a}$ \\
\hline$\left(\mathrm{UI} \mathrm{L}^{-1}\right)$ & GC & $308,8 \pm 115,9 \mathrm{a}$ & $382,0 \pm 135,5 b$ & $382,6 \pm 48,5 b$ & $433,2 \pm 61,3 b$ & $380,8 \pm 81,1 b$ & $297,8 \pm 85,8 \mathrm{a}$ \\
\hline CK & $\mathrm{GE} / \mathrm{Se}$ & $230,3 \pm 47,2 \mathrm{a}$ & $238,0 \pm 281,9 \mathrm{a}$ & $240,0 \pm 53,2 \mathrm{a}$ & $302,2 \pm 40,7 \mathrm{a}$ & $170,5 \pm 51,0 \mathrm{a}$ & $189,2 \pm 53,2 \mathrm{a}$ \\
\hline$\left(\mathrm{UI} \mathrm{L}^{-1}\right)$ & $\mathrm{GC}$ & $196,5 \pm 246,1 \mathrm{a}$ & $209,3 \pm 72,5 \mathrm{a}$ & $221,5 \pm 47,4 \mathrm{a}$ & $210.3 \pm 84,2 \mathrm{a}$ & $295.8 \pm 219,0 \mathrm{a}$ & $288.2 \pm 53,2 \mathrm{a}$ \\
\hline Glicose & $\mathrm{GE} / \mathrm{Se}$ & $89,5 \pm 4,5 \mathrm{a}$ & $83,8 \pm 10,5 \mathrm{a}$ & $89,0 \pm 4,2 \mathrm{a}$ & $83,5 \pm 8,9 \mathrm{a}$ & $85,7 \pm 9,7 \mathrm{a}$ & $93,3 \pm 5,3 \mathrm{a}$ \\
\hline$\left(m g \mathrm{dL}^{-1}\right)$ & GC & $95,3 \pm 14,4 \mathrm{a}$ & $98,8 \pm 10,9 \mathrm{a}$ & $95,6 \pm 7,9 \mathrm{a}$ & $86,3 \pm 5,5 \mathrm{a}$ & $90,0 \pm 5,8 \mathrm{a}$ & $93,0 \pm 7,4 \mathrm{a}$ \\
\hline Lactato & $\mathrm{GE} / \mathrm{Se}$ & $0,7 \pm 0,1 \mathrm{a}$ & $4,5 \pm 1,9 b$ & $6,0 \pm 2,9 b$ & $0,5 \pm 0,2 \mathrm{a}$ & $0,5 \pm 0,2 \mathrm{a}$ & $0,5 \pm 0,1 \mathrm{a}$ \\
\hline$\left(\mathrm{mmol} \mathrm{L}{ }^{-1}\right)$ & $\mathrm{GC}$ & $0,6 \pm 0,2 \mathrm{a}$ & $8,2 \pm 4,2 \mathrm{~b}$ & $6,5 \pm 3,8 \mathrm{c}$ & $0,6 \pm 0,1 \mathrm{a}$ & $0,7 \pm 0,2 \mathrm{a}$ & $0,5 \pm 0,1 \mathrm{a}$ \\
\hline CAT & $\mathrm{GE} / \mathrm{Se}$ & $664,9 \pm 16,9 \mathrm{a}$ & $629,4 \pm 21,6 b$ & $634,3 \pm 14,3 b$ & $660,5 \pm 15,3 \mathrm{ac}$ & $656,5 \pm 20,8 \mathrm{bc}$ & $673,3 \pm 23,0 \mathrm{~d}$ \\
\hline$\left(\mathrm{mmol} \mathrm{L}^{-1}\right)$ & GC & $665,2 \pm 14,0 \mathrm{ac}$ & $635,0 \pm 15,6 b$ & $644,6 \pm 18 b$ & $658,2 \pm 19,7 \mathrm{c}$ & $654,4 \pm 19,5 b c$ & $676,1 \pm 18,9 \mathrm{~d}$ \\
\hline
\end{tabular}

Médias e desvios-padrão seguidas das mesmas letras nas linhas não diferem estatisticamente entre si. Médias e desvios-padrão seguidos das mesmas letras maiúsculas na coluna ou sem elas não diferem estatisticamente entre si, $\alpha=0,05$. Zero $=$ momento 15 min antes do início do exercício. $\mathrm{AST}=$ aspartato aminotrasnferase; $\mathrm{LDH}=$ lactato desidrogenase; $\mathrm{CK}=$ criatinoquinase; $\mathrm{CAT}=$ capacidade antioxidante total.

somente valores maiores que $10.000 \mathrm{UI} \mathrm{L}^{-1}$ refletiriam lesão significante. A suplementação com vit-E e selênio não influenciou significativamente nesses indicadores. Outros autores obtiveram resultados semelhantes para equinos submetidos a exercícios de alta atividade e curta duração (SICILIANO et al., 1997; DIAS et al., 2009).

Os valores de glicemia não evidenciaram diferenças significativas entre os grupos, ou nos momentos avaliados (Tabela 1). Já, o pico de lactatemia foi de $7,1 \mathrm{mmol} \mathrm{L}^{-1}$ no GC após o primeiro chukka, enquanto, no GE/Se, foi de $6,5 \mathrm{mmol} \mathrm{L}^{-1}$ somente após o segundo chukka, contudo não houve diferença significativa entre os grupos $(\mathrm{P}=0,2571)$. As alterações observadas em relação ao repouso foram significativas $(\mathrm{P}=0,0002)$. Em ambos os grupos, o retorno aos valores semelhantes ao basal ocorreu $24 \mathrm{~h}$ após a partida. Esses resultados foram semelhantes aos descritos por HARKINS et al. (1993) e ZOBBA et al. (2011) em cavalos de polo e evidenciam a alta intensidade do trabalho executado. Valores maiores que $4 \mathrm{mmol} \mathrm{L}^{-1}$ indicam um esforço de alta intensidade e valores entre 2,5 e $4 \mathrm{mmol} \mathrm{L}^{-1}$ indicam esforço de intensidade moderada (SANTOS, 2006).

Observou-se redução significativa $\left(\mathrm{P}=2.90^{-25}\right)$ da CAT de 664,9mmol $\mathrm{L}^{-1} \quad(\mathrm{GE} /$ $\mathrm{Se})$ e $665,2 \mathrm{mmol} \mathrm{L}^{-1}(\mathrm{GC})$ antes do jogo para $629,4 \pm 21,6 \mathrm{mmol} \mathrm{L}^{-1}(\mathrm{GE} / \mathrm{Se})$ e $635,0 \pm 15,6 \mathrm{mmol}$ $\mathrm{L}^{-1}$ (GC) após os chukkas, retornando a valores semelhantes ao basal às $24 \mathrm{~h}$ e superando esses valores às $72 \mathrm{~h}\left(673,3 \pm 23,0 \mathrm{mmol} \mathrm{L}^{-1}\right.$ no GE/Se e $676,1 \pm 18,9 \mathrm{mmol} \mathrm{L}^{-1}$ no GC). Não foi observada diferença entre os grupos GE/Se e GC (Tabela 1).

A CAT no soro é o resultado das interações entre cada um dos componentes antioxidantes do soro. Essa interação pode proporcionar maior proteção contra os radicais livres do que qualquer antioxidante isoladamente (WAYNER et al. 1987). Antioxidantes relevantes são classificados em dois grupos: de prevenção, que impedem o início das reações em cadeia das ERO (Superoxido dismutase, catalase, glutationa peroxidase, transferina, ceruloplasmina e albumina) e os de interseptação, que podem prender os radicais livres diretamente, interrompendo assim reações em cadeia de propagação (glutationa, ácido úrico, ácido ascórbico, $\alpha$-tocoferol, carotenoides) (FERREIRA et al., 2008).

Estudos sobre a CAT em equinos são ainda restritos. EL-DEEB \& EL-BAHR (2010) relataram redução da CAT em cavalos Árabes, acometidos por rabdomiólise $\left(0,23 \pm 0,12 \mathrm{nmol} \mathrm{L}^{-1}\right)$ contra $\left(0,53 \pm 0,39 \mathrm{nmol} \mathrm{L}^{-1}\right)$ em cavalos hígidos. EL-ASHKER (2011a) verificou valores de CAT de $0,79 \pm 0,05 \mathrm{nmol} \mathrm{L}^{-1}$ para cavalos hígidos, $0,72 \pm 0,04 \mathrm{nmol} \mathrm{L}^{-1}$ para cavalos com rabdomiólise moderada e $0,27 \pm 0,04 \mathrm{nmol} \mathrm{L}^{-1}$ com rabdomiólise severa. El-ASHKER (2011b) relatou que cavalos árabes de tração que morreram em decorrência da

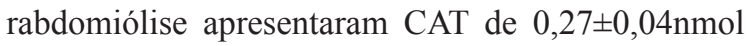
$\mathrm{L}^{-1}$. Os valores de CAT obtidos neste trabalho foram 
semelhantes aos relatados para cavalos hígidos (ELDEEB \& EL-BAHR, 2010; El-ASHKER, 2011-a e El-ASHKER 2011b). O comportamento observado de redução da CAT após o exercício e retorno aos valores normais após o repouso foi relatado também por BALOGH et al. (2001), quando observaram $0,73 \pm 0,04 \mathrm{nmol} \mathrm{L}^{-1}$ antes do exercício, $0,34 \pm 0,02 \mathrm{mmol}$ $\mathrm{L}^{-1}$ após o exercício e $0,53 \pm 0,24,24 \mathrm{~h}$ após o exercício. Essa redução se deve a um possível consumo dos agentes antioxidantes durante o exercício.

\section{CONCLUSÃO}

O exercício promoveu alterações bioquímicas e antioxidativas em cavalos submetidos à partida de polo e a suplementação com vitamina $\mathrm{E}$ e selênio não contribuiu para alterar esses parâmetros. $\mathrm{O}$ condicionamento físico dos animais estudados foi adequado para o esforço exigido em um jogo de polo.

\section{AGRADECIMENTOS}

À Fundação de Amparo à Pesquisa do Estado do Rio de Janeiro (Faperj), pelo apoio financeiro a este projeto.

\section{FONTES DE AQUISIÇÃO}

a - Corcelina - Agribands Purina.

b - Purinafos 80 - Agribands Purina.

c - E-S-E Super- Vetnil Ind. e Com. de Produtos Veterinários Ltda.

d - Analisador Hematológico Veterinário pocH-100iV Diff da Sysmex.

e - Kit para determinação de Lactato - Katal Biotecnológica Ind. e Com. Ltda.

f - BR.A15: Biosystems.

g - Antioxidant Assay Kit - SIGMA - referência CS0790.

$\mathrm{h}$ - Thermo Plate modelo TP Reader.

\section{COMITÊ DE ÉTICA}

Protocolo 161/11 - UFRRJ.

\section{REFERÊNCIAS}

ARAYA, O. et al. Efecto del selenato de bario inyectable sobre la actividad de glutation peroxidasa en caballos a pastoreo. Arch med vet, v.36, n.1, p. 31-37, 2004. Disponível em: <http://www. scielo.cl/scielo.php?script=sciarttext\&pid $=$ S0301732X200400010 0003\&lng=es\&nrm=iso>. Acesso em: 18 jan. 2011. doi: $10.4067 /$ S0301-732X2004000100003.

BALOGH, N. et al. Biochemical and antioxidant changes in plasma and erythrocytes of pentathlon horses before and after exercise. Vet Clin Path, v.30, n.4, p.214-218, 20000001. Disponível em: <http://www.doi.com/10.1111/j.1939165X.2001. tb00434.x/pdf>. Acesso em: 16 out. 2011. doi/ 10.1111/j.1939165X.2001.tb00434.x.

CAIADO, J.C.C. et al. Lactacidemia e concentrações séricas de aspartato aminotransferase e creatinoquinase em equinos da raça quarto de milha usados em provas de laço em dupla. Pesq Vet Bras, v.31, n.5, p.452-458, 2011. Disponivel em: <http://dx.doi. org/10.1590/S0100-736X2011000500014>. Acesso em: 16 out. 2011. doi:10.1590/S0100-736X2011000500014.

CORREA, K.S. et al. Enzimas musculares e eletrólitos em eqüinos submetidos a esforço físico prolongado, suplementados com acetato de tocoferol e selênio. Vet Zoot, v.17, n.1, p.8593, 2010. Disponível em: <http://www.cabi.org/cabdirect/ FullTextPDF/2010/20103218462.pdf>. Acesso em: 28 dez. 2010.

DEATON, C.H.M.; MARLIN, D.J. Exercise associated oxidative stress. Clin Tech in Eq Pract, v.2, p.278-291, 2003. Disponível em: <http://dx.doi.org/10.1053/S1534-7516(03)00070-2>. Acesso em: 17 out. 2011. doi/10.1053/S1534-7516(03)00070-2.

DIAS, D. et al. Efeito da suplementação com vit-E/ Se sobre o quadro hematológico, enzimas marcadoras de lesão muscular e índice de peroxidação de biomoléculas em equinos submetidos à atividade de salto. Ciên Anim Bras, v.10 n.3, p.790-801, 2009. Disponível em: <http://www.revistas.ufg.br/index.php/vet/article/ view/4793>. Acesso em: 16 out. 2011.

DUBERSTEIN, K.J. et al. Effects of vitamin E supplementation and training on oxidative stress parameters measured in exercising horses. Comp Ex Physiol, v.6, n.1, p.17-25, 2009. Disponível em: $<$ http://journals.cambridge.org/abstract_S1478061509341353>. Acesso em: 20 ago. 2012. doi:10.1017/S1478061509341353.

EL-ASHKER, M. Diagnostic efficiency of selected biochemical variables to predict the clinical outcome of exertional rhabdomyolysis in Egyptian draft horses. Comp Clin Pat, p.1-6, 2011a. Disponível em: <http://dx.doi.org/10.1007/s00580-011-1240-5>. Acesso em: 16 out. 2011. doi:10.1007/s00580-011-1240-5.

EL-ASHKER, M. Acute kidney injury mediated by oxidative stress in Egyptian horses with exertional rhabdomyolysis. Vet Res Comm Bio and L Sci, v.35, n.5 p.311-320, 2011b. Disponível em: $<\mathrm{http}$ ://dx.doi.org/10.1007/s11259-011-9475-9>. Acesso em: 17 out. 2011. doi: 10.1007/s11259-011-9475-9.

EL-DEEB, W.M.;EL-BAHR, S.M. Investigation of selected biochemical indicators of Equine rabdomyolysis in Arabian horses: pro-inflammatory cytokines and oxidative stress markers. Vet Res Comm, v.34, n.8, p.677-689, 2010. Disponível em: <http://www. springerlink.com/content/e81342nj84050113/>. Acesso em: 16 out. 2011. doi: 10.1007/s11259-010-9439-5.

FERREIRA, F. et al. Stress oxidativo e dano oxidativo muscular esquelético: influência do exercício agudo e treino físico. Rev Port Cien Desp, v.7, n.2, p.257-275, 2008. Disponível em: <http://www.scielo.oces. mctes.pt/pdf/rpcd/v7n2/v7n2a14.pdf>. Acesso em: 16 out. 2011.

FRANCINATO, C. et al. Atividade sérica das enzimas AST, CK e GGT em cavalos Crioulos. Pesq agropec bras, v.41, n.10, p.15611565, 2006. Disponível em: <http://dx.doi.org/10.1590/S0100204X2006001000014.A>. Acesso em: 16 out. 2011. doi: 10.1590/ S0100-204X2006001000014.

HARGREAVES, B.J. et al. Antioxidant status of horses during Two 80-km endurance races. J Nutr, v.132, n.6, p.1781-1783, 2002. Disponível em: $<$ http://jn.nutrition.org/content/132/6/1781S.full >. Acesso em: 16 out. 2011.

HARKINS, J.D. et al. Effect of detraining on indices of performance and their correlation with athletic ability of polo horses. J Equine 
Vet Sci, v.13, p.348-354, 1993. Disponível em: <http://www. sciencedirect.com/science/article/pii/S0737080606811238>. Acesso em: 03 jun. 2013.

HENNEKE, D.R. et al. Relationship between condition score, physical measurement, and body fat percentage in mares. Eq Vet J, v.15, p.371-372, 1983 .

KOBAYASHI, M. Simple lactate measurement in horses using a portable lactate analyzer with lancet skin punctures under field conditions. J Eq Sci, v.18, n.1, p.5-11, 2007. Disponível em: <http:// www.jstage.jst.go.jp/article/jes/18/1/5/_pdf.> Acesso em: 16 out. 2011.

KOWAL, R.J. et al. Avaliação dos valores de lactato e da atividade sérica da enzima creatina quinase em cavalos da raça PSI submetidos a teste de esforço em esteira ergométrica. $\mathbf{R}$ bras Ci Vet, v.13, n.1, p.13-19, 2006. Disponível em: <http://www.uff. br/rbcv/site/index.php/pages/ process?file=Artigo/95/arquivo_03. pdf $>$. Acesso em: 16 out. 2011

LIU, J. et al. Chronically and acutely exercised rats: biomarkers of oxidative stress and endoge-nous antioxidants. J Appl Physiol, n.89, p.21-28, 2000. Disponível em: <http://jap.physiology.org/ content/89/1/21.full.pdf $>$. Acesso em: 16 out. 2011

MICHIMA, L.E.S. et al. Estudo da isoenzima creatina quinase CKMB sérica em equinos de enduro após exercício físico prolongado. Braz J Vet Res AnimSci, v.47, n.1, p.23-30, 2010. Disponível em: $<$ http://www.revistasuspsibi.usp.br/scielo.php?script=sciarttext\&pid= $141395962010000100003 \& \ln =$ pt\&nrm=iso $>$. Acesso em: 16 out. 2011.

MOORE, R.M; KOHN, C.W. Nutritional muscular dystrophy in foals. Comp Cont Educ Pract Vet, v.13, p.476-489, 1991.

MOTA, M.P. et al. Teorias biológicas do envelhecimento. Rev Port Ciênc Desp,. v4, n.1, p.81-110, 2004. Disponível em: $<$ http://www.fade.up.pt/rpcd/arquivo/artigos_soltos/ol.4_nr.1/ Paula_Mota.pdf $>$. Acesso em: 17 out. 2011.

NATIONAL RESEARCH COUNCIL (NRC). Nutrient requirements of horses. 5.ed. Washington: National Academy of Science, 1989. 100p.

RIBEIRO, C.R. et al. Avaliação de constituintes séricos em eqüinos e muares submetidos à prova de resistência de $76 \mathrm{~km}$, no Pantanal do Mato Grosso, Brasil. Cienc Rural, v.34, n.4, p.10811086, 2004. Disponível em: <http://dx.doi.org/10.1590/S0103$84782004000400018>$. Acesso em: 16 out. 2011. doi: 10.1590 S0103-84782004000400018

RICH, G.A.; BREUER, L.H. Recent developments in equine nutrition with farm and clinic applications in depth: current concepts in equine nutrition. Proc Ann Conv AAEP, v.48, p.22-40, 2002. Acesso em: 11 ago. 2012. Online. Disponível em: $<$ http://www.ivis. org/proceedings/AAEP/2002/910102000024.PDF>.

RICHARDSON, S.M. et al. Effect of selenium supplementation and source on the selenium status of horses. J Anim Sci, v.84, n.7, p.1742-1748, 2006. Disponível em: <http://www.ncbi.nlm.nih. gov/pubmed/16775058>. Acesso em: 3 jun. 2013.

SANTOS, V.P. Variações hemato-bioquímicas em equinos de salto submetidos a diferentes tipos de protocolos de exercício. 2006. 94f. Dissertação (Mestrado em Medicina Veterinária) Curso de Pós-graduação em Ciências Veterinárias, UFRGS, Porto Alegre, RS. Disponível em <http://hdl.handle.net/10183/6797>. Acesso em: 10 ago. 2012.

SICILIANO, P.D. et al. Effect of dietary vitamin E supplementation on the integrity of skeletal muscle in exercised horses. J Anim Sci, v.75, n.6, p.1553-1560, 1997. Disponível em: <http://jas.fass.org/ content/75/6/1553>. Acesso em: 17 out. 2011

THOMASSIAN, A. et al. Atividades séricas da aspartato aminotransferase, Creatino-quinase e lactato desidrogenase de eqüinos submetidos ao teste padrão de exercício.progressivo em esteira. Braz J Vet Res Ani Sci, v.44, n.3, p.183-190, 2007. Disponível em: <http:// www.revistas.usp.br/bjvras/article/download/26637/28420>. Acesso em: 03 jun. 2013.

VAN DEN HOVEN, R. et al. A preliminary study on the changes in some potential markers of muscle-cell degradation in submaximally exercised horses supplemented with a protein and amino acid mixture. J An Phys and An Nut, v.95, n.5, p.664675, 2011. Disponível em: <http:/www.ncbi.nlm.nih.gov/ pubmed/21121963>. Acesso em: 3 jun. 2013.

VOLFINGER, L. et al. Kinetic evaluation of muscle damage during exercise by calculation of amount of creatine kinase released. Am J of Phys, v.266, p.434-341, 1994. Disponível em: <http://ajpregu. physiology.org/content/266/2/R434.full.pdf>. Acesso em: 17 out. 2011.

WAYNER, D.D.M. et al. The relative contributions of vitamin E, urate, ascorbate and proteins to the total peroxyl radical-trapping antioxidant activity of human blood plasma. Biochim Biophys Acta, v.924, p.408-419, $1987 . \quad$ Disponível em: <http://www. sciencedirect.com/science/ article/pii/0304416587901553>. Acesso em: 24 out. 2011. doi:10.1016/0304-4165(87)90155-3.

WILLIAMS, C.A. et al. Oxidative stress in horses in three $80 \mathrm{~km}$ races. Eq. Nutr Phys Soc Proc, v.18, p.47-52, 2003. Disponível em: <http://scholar.lib.vt.edu/theses/available/etd-06262003-095741/ unrestricted/paper3.pdf>. Acesso em: 17 out. 2011.

YONEZAWA, L.A. et al. Malondialdeído e troponina I cardíaca em equinos da raça Puro Sangue Árabe submetidos ao exercício e à suplementação com vitamina E. Cienc Rural, v.40, n.6, p.13211326, 2010. Disponível em: <http://www.scielo.br/pdf/cr/v40n6/ a613cr2968.pdf>. Acesso em: 03 jun. 2013. doi:10.1590/S010384782010005000094 .

ZOBBA, R. et al. Physical, hematological, and biochemical responses to acute intense exercise in polo horses. J Equine Vet Sci, v.31, n.9, p.542-548, 2011. Disponível em: <http://www. sciencedirect.com/science/article/pii/S073708061100102X $>$. Acesso em: 03 jun de 2013. 